\title{
OBTENÇÃO E CARACTERIZAÇÃO DE ESPUMAS RÍGIDAS DE POLIURETANO (PUR) COM PÓ DE VIDRO SODO-CÁLCICO (PV) DO REJEITO DA LAPIDAÇÃO
}

\author{
Á. C. P. GALVÃO*, A. C. M. FARIAS e J. U. L. MENDES \\ Universidade Federal do Rio Grande de Norte - UFRN \\ galvao_alvaro@hotmail.com*
}

Artigo submetido em abril/2014 e aceito em setembro/2015

DOI: $10.15628 /$ holos.2015.2085

\section{RESUMO}

O isolamento térmico é empregado na proteção de superfícies aquecidas ou resfriadas através de materiais de baixa condutividade térmica, onde as espumas rígidas de poliuretano (PUR) são as mais utilizadas. $\mathrm{O}$ rejeito de vidro não é aproveitável pela indústria recicladora, por isto algumas pesquisas buscam um aproveitamento desse resíduo vítreo aplicando-o como reforço em materiais. Neste trabalho foram desenvolvidos compósitos de PUR carregadas com pó de vidro (PV) refinado objetivando uma comparação com o PUR puro e sua adequação como isolante térmico. O PV foi triturado e moído, obtendo-se duas granulometrias diferentes, as quais foram caracterizadas por Análise de Granulometria a Laser, DRX e FRX. Os corpos-de-prova de PUR+PV foram produzidos aplicando-se percentuais mássicos de PV a 5, 10 e 20, cujas características foram analisadas através de ensaios de condutividade térmica, massa específica e resistência à compressão. Os resultados demonstraram que as propriedades dos compósitos de PUR são influenciadas pelos percentuais de PV adicionados na matriz de PUR. Assim, constatouse que a resistência à compressão (exceto o PV20-100) e a massa específica dos compósitos PUR+PV foi superior à do PUR puro. A condutividade térmica dos compósitos PUR+PV permaneceu numa faixa de 0,035 a 0,045 W/m$K$, o que pode sugerir que a inclusão do $P V$ não afetou significativamente as propriedades termofísicas, quando comparadas às do PUR puro $(0,040 \mathrm{~W} / \mathrm{m}-\mathrm{K})$. A partir destas análises, a aplicabilidade do rejeito vítreo contribuiu como reforço do PUR e a sua função de isolação térmica não foi reduzida. Isto possibilita maior economia e preservação ambiental.

PALAVRAS-CHAVE: compósitos, espuma PUR, pó de vidro, condutividade térmica, resistência à compressão.

\section{OBTAINING AND CHARACTERIZATION OF THE RIGID POLYURETHANE FOAMS (PUR) FILLED WITH SODA-LIME GLASS POWDER (GP) FROM WASTE OF LAPPING}

\begin{abstract}
Thermal insulation is used in the protection of heated or cooled surfaces by the low thermal conductivity materials, where the rigid polyurethane foams (PUR) are the most widely applied. Glass wastes are not usable by the recycling industry, so some researches looking for a harnessing of this glassy residue to apply it as reinforcement materials. In this paper were developed PUR composites filled with refined glass powder (GP) to compare with pure PUR and to evaluate their suitability as thermal insulators. PV was milled and sifted to obtain two different particle sizes. These particles were characterized by laser granulometry, XRD and XRF analyses. The PUR+PV samples were produced by application of PV of 5, 10 and 20 percentage mass. The characteristics of samples were analyzed by the
\end{abstract}

thermal conductivity, density and compressive strength tests. The results showed the properties of PUR composites are influenced by the addition of PV on PUR matrix. Thus, it was found that the compression strength (except to PV20-100) and the density of the PUR + PV composites were higher than that of pure PUR. The thermal conductivity of the PUR + PV composites showed a range of 0.035 until $0.045 \mathrm{~W} / \mathrm{m}-\mathrm{K}$. This can suggest that the inclusion of PV didn't affect significantly the thermophysical properties when they were compared to pure PUR $(0.040 \mathrm{~W} / \mathrm{m}-\mathrm{K})$. Therefore, the applicability of the glass waste contributed to improve the PUR strength, and also its thermal insulation was not reduced. This can allow high economy and environmental preservation.

KEYWORDS: composite, PUR foam, glass powder, thermal conductivity, compressive strength 


\section{INTRODUÇÃO}

Segundo a ABQUIM (2010), o poliuretano (PU) ainda é visto como um produto caro, apesar de seus benefícios e durabilidade no médio e longo prazo quando comparado com seus concorrentes, como, por exemplo, o isopor (EPS). O poliuretano PU é composto por mais de $90 \%$ de ar e o restante de plástico e, portanto, economicamente inviável para o reaproveitamento como matéria-prima. $\mathrm{O}$ crescente uso desse material trouxe como consequência a preocupação com o destino final dos refugos, aparas e rebarbas, provenientes dos processos industriais (Siqueira e Stramari, 2004).

Além disso, a incineração consiste na combustão do lixo, reduzindo o volume deste em aterros. Porém possui um custo bastante elevado e, em alguns casos, pode ser de alto risco visto que certos materiais podem liberar gases nocivos à saúde da população (Cangemi, 2006). Devido às propriedades físico-químicas da espuma do PU, esse material se torna demasiadamente caro para o armazenamento em aterro, o que se justifica pelo grande volume ocupado (baixa densidade) e seu longo tempo de decomposição de $\approx 150$ anos (Fischer, 2002 e Siqueira e Stramari, 2004). Como as matérias-primas da espuma são derivadas do petróleo, a não reciclagem da espuma também representa um desperdício de recursos naturais não renováveis (Mello et al., 2006). Deste modo, as empresas tem feito uma restrição ao conceito de crescimento e passaram a introduzir a questão ecológica como critério fundamental das atividades de negócio.

A porosidade dos materiais pode influenciar na resistência à compressão e na condutividade térmica. Estes materiais porosos incluem as espumas, as quais são influenciadas pela estrutura/ morfologia de poros resultando na sua baixa densidade. Geralmente, materiais com elevada porosidade e pequena estrutura de poro celular regular apresentam as melhores características para obter espumas (Vladimirov et al., 2011).

A matéria-prima tradicional para a fabricação da espuma rígida de poliuretano, a PUR, o poliol e isocianato, pode ser reduzida através da combinação/inclusão de outros produtos/cargas (Vilar, 2007). Santos (2009) defendeu o reaproveitamento de entulhos de vidro, ou outro material inorgânico como material de adição à matriz de materiais mais caros com fins de viabilizá-los ecológico e economicamente. Segundo ele, o uso vidro descartado pode oferecer uma série de vantagens como: diminuição de custos de coleta, redução da poluição ambiental, aquecimento da economia e redução do consumo de recursos naturais.

O vidro é um material inerte não cristalino, não poroso e frágil, e também é considerado um isolante térmico. Por apresentar estas características e ser impermeável à passagem de oxigênio ou gás carbônico, o aproveitamento do vidro de entulhos, como carga de enchimento na confecção de PUR, pode oferecer uma série de vantagens como: diminuição de custos de coleta, redução da poluição ambiental, aquecimento da economia e redução do consumo de recursos naturais (Santos, 2009).

Santos (2009) estudou o uso de vidro na produção de novos materiais compósitos e concluiu, através de seu trabalho sobre sinterização de pó de vidro (PV), que, conforme o tamanho médio da partícula era reduzido $(98,6 \mu \mathrm{m} ; 30,7 \mu \mathrm{m} ; 14,9 \mu \mathrm{m})$, maior era a sua reatividade química devido ao aumento da energia superficial. A partir desta análise, ele observou que a temperatura inicial de retração, durante o processo de sinterização, era 
diretamente proporcional ao tamanho desses pós. Consequentemente, haveria diminuição do consumo de energia e impacto ambiental causado pela geração de gases.

Tsai et al. (2007) desenvolveram um material compósito de PUR com fibras ocas de poliéster (PET) tratadas com retardante de chama. No processo de preparação do compósito, o PU reagiu e expandiu entre duas mantas de poliéster, conformado em um molde fechado. Noutro estudo, Bledzki et al., (2001) apud Yang et al. (2004) avaliaram o efeito da adição de fibras e/ou tecidos em PUR e concluíram que, encontrando um conteúdo ideal de fibras, a resistência mecânica, a dureza e a resistência ao impacto da espuma aumentaram.

As propriedades da espuma de PU podem ser manipuladas para atender diferentes requisitos de aplicação: flexível, rígido, plástico, elástico ou termofixo, compacto ou em espuma (Demharter, 1998). As PUR são muito usuais devido a sua grande faixa de propriedades, principalmente como materiais estruturais (resistência mecânica e alta tenacidade) e isolação térmica. As propriedades da PUR são fortemente influenciadas pela estrutura, geometria e massa específica da espuma, bem como a adição, tamanho e formas das cargas na PUR (Thirumal et al., 2007).

Saha et al. (2008) estudaram os efeitos no desempenho térmico e mecânico da PUR (derivada de petróleo) causados pela adição de três tipos de nanopartículas $\left(\mathrm{TiO}_{2}\right.$, nanoargila e nanofibra de carbono, CNF). Eles observaram uma melhora significativa nas propriedades do material com a adição destas nanopartículas. A PUR/CNF apresentou os melhores resultados, e o $\mathrm{PUR} / \mathrm{TiO}_{2}$, os piores desempenhos em todos os ensaios realizados. Com a adição de apenas $1 \%$ de CNF houve um aumento de $86 \%$ no módulo de elasticidade da PUR, $40 \%$ na resistência à compressão e $45 \%$ na flexão, além do aumento na temperatura de decomposição em $18{ }^{\circ} \mathrm{C}$.

Thirumal et al. (2008) analisaram a adição de grafite expansível (EG) com volumes mássicos de 5 a $50 \%$ wa matriz da PUR, cujas partículas mediam de 180 e $300 \mu \mathrm{m}$. Eles verificaram que, com o aumento das proporções de EG, diminuía-se a resistência à compressão. As PUR carregadas com partículas maiores de EG obtiveram melhores resultados. Isto foi explicado pelo fato de que os grãos não se localizavam na estrutura da célula, porque as partículas menores foram mal dispersadas e formaram agregados. Isto levou a uma estrutura celular heterogênea, resultando em baixas propriedades mecânicas.

Torreira (1980) afirma que para ser considerado um bom isolante térmico, o material deve apresentar as seguintes características: baixa condutividade térmica; boa resistência mecânica; baixa massa específica; baixa difusividade térmica; alto calor específico; resistência à combustão; baixo coeficiente de expansão térmica; estabilidades química e física; resistência específica ao ambiente de utilização; baixa higroscopicidade; ausência de odor e baixo custo. A função primária de um isolante térmico é reduzir a taxa de transferência de calor entre um sistema e o meio, de modo que a energia possa ser conservada. Para isso, partes dos sistemas térmicos devem ser revestidas com material que seja estável a um determinado intervalo de temperatura [Torreira (1980) e Mendes (2002)]. A eficiência isolante de um material varia na razão inversa do seu peso específico (Rapin, 2001), como as espumas, por exemplo.

De acordo com Oliveira (2010), as propriedades térmicas e acústicas da espuma de PUR estão relacionadas à sua microestrutura. As PUR possuem uma microestrutura com células fechadas, que confinam o ar em seu interior, enquanto os poliuretanos flexíveis são compostos de células abertas que permitem o fluxo de ar entre seus poros. De acordo com Kipper (2008), as 
espumas rígidas possuem estrutura rígida altamente reticulada e com células fechadas, responsável pelas suas propriedades mecânicas. Além disso, a condutividade térmica do gás (ar) retido nestas células fechadas é o fator preponderante nas propriedades isolantes da espuma, visto que $\mathrm{o}$ ar é reconhecido como um excelente isolante térmico. Ainda considera-se a espessura desse material como um dos principais fatores que afetam a condutividade térmica dos isolantes; outros fatores incluem a massa especifica e tamanho das células do material, umidade e temperatura ambiente (Mendes, 2002).

Este trabalho objetiva investigar a influência do tamanho das partículas de pó de vidro PV $(100 \mu \mathrm{m}$ e $300 \mu \mathrm{m})$ e o seu percentual mássico (5, 10 e $20 \%)$ na matriz de espuma rígida de mamona - PUR através de ensaios de condutividade térmica, massa específica e resistência à compressão dos corpos-de-prova de PUR puro (padrão de referência) e de PUR+PV. Além disto, objetiva-se também verificar a se há correlação entre massa específica e condutividade térmica dos materiais estudados.

\section{METODOLOGIA}

Este trabalho tratou do estudo da fabricação, realização de ensaios e análises de sete tipos de formulações de compósitos de PUR e PV e PUR puro, usando-se cinco corpos-de-prova de cada formulação. As propriedades destes materiais foram avaliadas e comparadas através dos ensaios de condutividade térmica, resistência à compressão e massa específica dos corpos-deprova desenvolvidos. As caracterizações granulométrica e química do PV também foram investigadas. Estes ensaios foram executados nos Laboratórios de Mecânica dos Fluidos, Ensaios de Materiais do NTI da UFRN. A Figura 1 apresenta o fluxograma desde a obtenção dos materiais até a execução de ensaios e análises realizados.

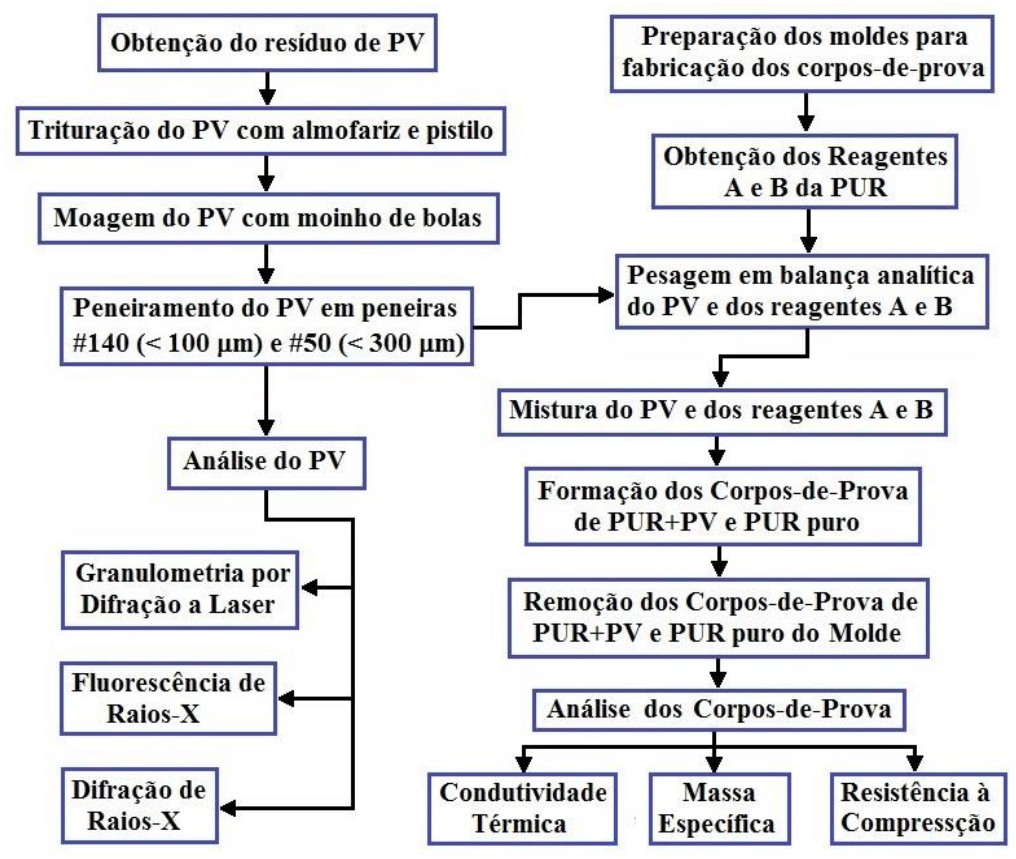

Figura 1 - Fluxograma de preparação dos corpos-de-prova, realização de ensaios e análises.

Os reagentes de $\mathrm{A}$ e $\mathrm{B}$ de $\mathrm{PU}$, adquirido comercialmente, foi originário do petróleo, enquanto que o PV (rejeito do processo de lapidação de vidro) foi cedido pela DVN Vidros LTDA de Natal/RN. 


\subsection{Caracterização do Resíduo Pó de Vidro}

A caracterização do resíduo de pó de vidro PV proveniente da indústria de vidrarias visou proporcionar alternativas tecnológicas e ambientais para a reciclagem dos mesmos através do estudo das propriedades espectroscópicas e morfológicas deste resíduo. Sendo assim, esse material foi caracterizado por distribuição granulométrica e pelas técnicas de Fluorescência de raios-X (FRX) e Difração de raios-X (DRX).

O pó de vidro foi peneirado e a porção selecionada foi quantidade passante nas peneiras de malha 50 ABNT $(<300 \mu \mathrm{m})$ e 140 ABNT $(<100 \mu \mathrm{m})$. A distribuição granulométrica foi realizada pela técnica de classificação de partículas por difração a laser. O equipamento utilizado foi um Granulômetro a laser, modelo CILAS $1180(0,04 \mu \mathrm{m}-2.500 \mu \mathrm{m})$ do Laboratório de Técnicas Mineral e de Materiais/NIT/UFRN.

A análise química do pó de vidro foi realizada por fluorescência de raios $\mathrm{X}$ pelo método do semiquantitativo, em forma de pó, sob atmosfera a vácuo, colimador $10 \mathrm{~mm}$, cujo equipamento utilizado foi o modelo XFR-1800 marca SHIMADZU pertencente ao Laboratório de NUP-ER (Núcleo de Estudos em Petróleo e Energias Renováveis) (CCET/UFRN).

$A$ análise de difração de raios $X$ foi realizada em um equipamento difratômetro de raios $X$ da SHIMADZU, modelo XRD-7000 com radiação de CuK $\alpha$ e comprimento de onda $\lambda=1,5406 \AA$, que opera a uma voltagem de $40 \mathrm{KV}$ com $30 \mathrm{~mA}$ de corrente. A amostra em forma de pó (\#50) foi analisada em um intervalo de varredura entre 10 e 90 graus, a uma taxa de $2 \circ \mathrm{C} / \mathrm{min}$. $O$ ensaio foi realizado no Laboratório de Engenharia de Materiais (NIT/UFRN)

\subsection{Confecção dos Corpos-de-Prova}

A mistura de PUR e PV foi conformada em moldes retangulares $(10 \mathrm{~cm} \times 7 \mathrm{~cm} \times 8 \mathrm{~cm})$, cujo processo de fabricação dos corpos-de-prova seguiu as seguintes etapas:

1- Trituração do resíduo de vidro, com o auxílio de pistilo e almofariz.

2- Uso de moinho de bolas com esferas de alumina para reduzir a granulometria.

3- Peneiramento manual para obtenção de dois de tamanhos de partículas.

4- Análise e classificação do tamanho médio das partículas por granulômetro a laser.

5- Preparação dos moldes retangulares $(10 \mathrm{~cm} \times 7 \mathrm{~cm} \times 8 \mathrm{~cm})$ para fabricação dos corpos-de-prova.

6- Pesagem dos reagentes A-Poliol e B-Isocianato (proporção mássica de 1:1) e do PV para obtenção dos corpos-de-prova de PUR + PV, usando-se uma balança de precisão Bel Engineering, capacidade máxima $1000 \mathrm{~g} \pm 0,001 \mathrm{~g}$, disponível no LMF do NTI/UFRN.

7- Mistura dos reagentes no molde.

8- Formação dos corpos-de-prova.

9- Remoção dos corpos-de-prova dos moldes.

Na Figura 2 são ilustrados os processos de obtenção do PV e da fabricação dos corpos-deprova, isto é, a obtenção das PUR com adição de pó de vidro. Os corpos-de-prova de PUR preenchidos com PV e o de PUR (padrão de referência) estão classificados na Tabela 1, indicando os percentuais mássicos e o tamanho das partículas de PV usados. O tempo de cura foi estabelecido em 48 horas, conforme norma NBR 8082/83, antes de eles serem submetidos a ensaios de resistência à compressão e de condutividade térmica. 

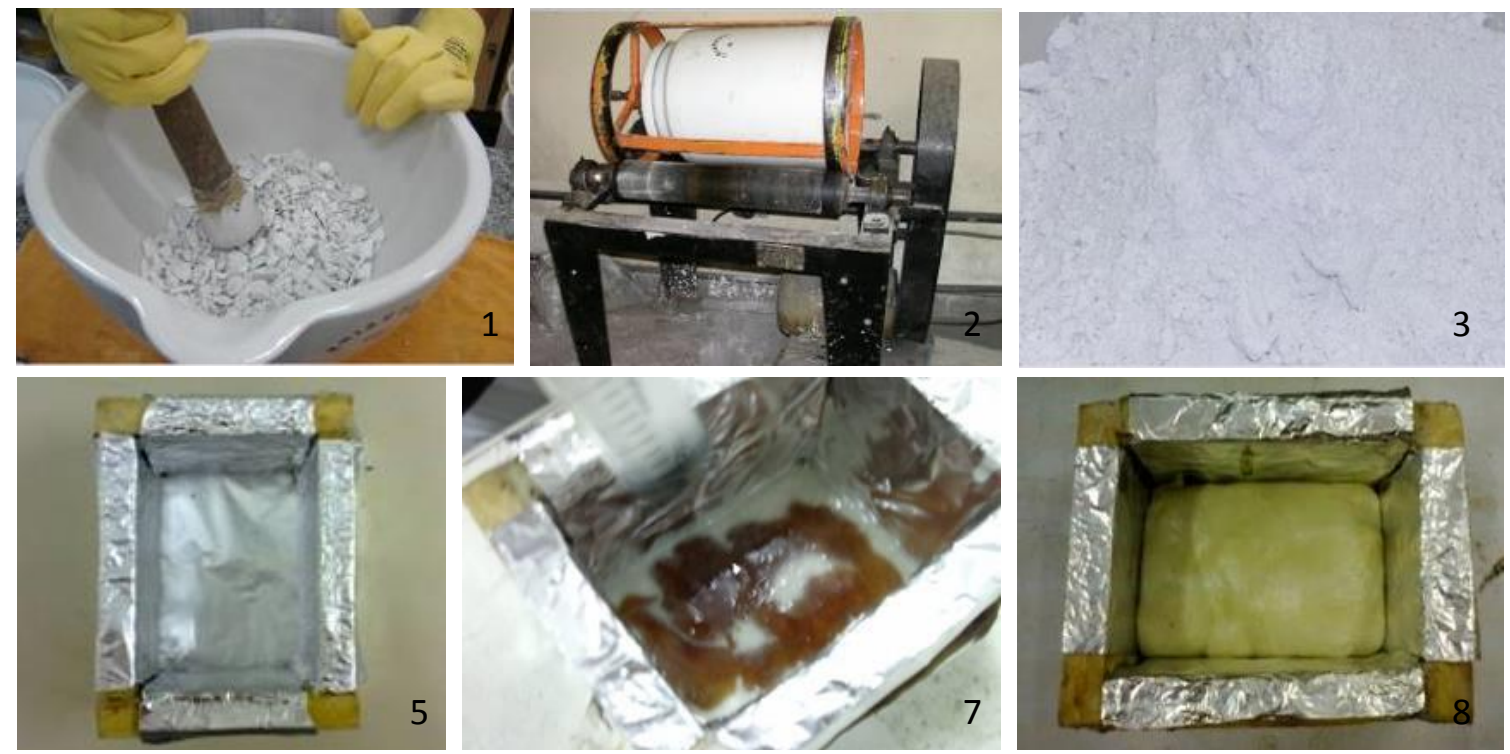

Figura 2 - Imagens das principais etapas do processo de obtenção dos corpos-de-prova de PUR+PV.

Tabela 1: Descrição dos corpos-de-prova.

\begin{tabular}{l|l}
\hline Corpos-de-Prova & \multicolumn{1}{c}{ Descrição } \\
\hline PUR-puro & PUR sem adição de carga de PV \\
\hline PV5-100 & PUR com carga de $5 \%$ de PV, partículas de até $100 \mu \mathrm{m}$ \\
\hline PV5-300 & PUR com carga de $5 \%$ de PV, partículas de até $300 \mu \mathrm{m}$ \\
\hline PV10-100 & PUR com carga de $10 \%$ de PV, partículas de até $100 \mu \mathrm{m}$ \\
\hline PV10-300 & PUR com carga de $10 \%$ de PV, partículas de até $300 \mu \mathrm{m}$ \\
\hline PV20-100 & PUR com carga de $20 \%$ de PV, partículas de até $100 \mu \mathrm{m}$ \\
\hline PV20-300 & PUR com carga de $20 \%$ de PV, partículas de até $300 \mu \mathrm{m}$ \\
\hline
\end{tabular}

\subsection{Ensaio de Resistência à Compressão}

A resistência mecânica dos corpos-de-prova de PUR foi avaliada através de ensaios de resistência à compressão, usando uma máquina de ensaio universal SHIMADZU, modelo AUTOGRAPH AG-X 300KN, com capacidade de compressão de 284 KN, disponível no Laboratório de Ensaios Mecânicos do NTI/UFRN.

O ensaio de resistência à compressão seguiu os procedimentos da norma NBR 8082/83. Após a aplicação da pré-carga de $5 \mathrm{~N}$, a velocidade de aplicação de carga compressiva foi mantida em $0,25 \mathrm{~cm} / \mathrm{min}$. O critério de final de ensaio foi determinado pela redução de $10 \%$ na deformação longitudinal do corpo-de-prova, conforme visto na Fig. 3.

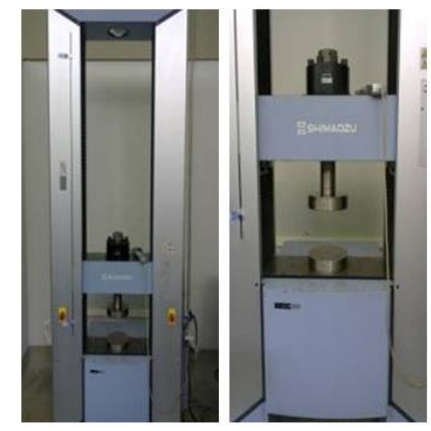

(a)

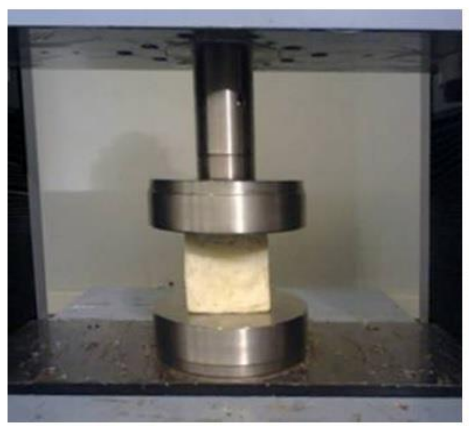

(b)

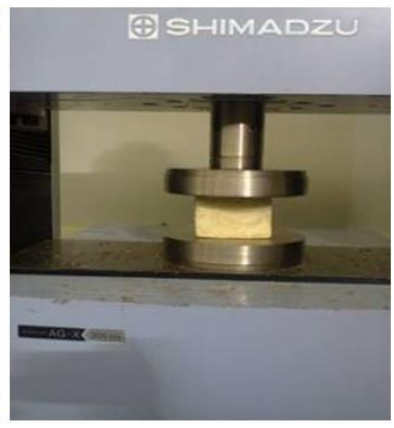

(c)

Figura 3 - Ensaio de compressão: (a) montagem, (b) pré- carga aplicada e (c) material comprimido. 
Os resultados dos ensaios foram armazenados na forma de gráficos de Tensão $\mathrm{x}$ Deformação, calculados de acordo com a Eq. 1:

$R_{C}=\frac{F}{A}$

em que :

$R_{c}=$ resistência à compressão a $10 \%$ de deformação $(\mathrm{Pa})$;

$F=$ força máxima anotada $(\mathrm{N})$;

$A=$ área do corpo de prova $\left(\mathrm{m}^{2}\right)$.

\subsection{Ensaio de condutividade térmica}

No ensaio de condutividade térmica utilizou-se o equipamento KD2 Pro, disponível no Laboratório de Mecânica dos Fluidos do NTI/UFRN. Este equipamento serve para efetuar medição de propriedades térmicas na faixa de 0,02 a $2,00 \mathrm{~W} / \mathrm{mK}$ e possui um sensor $\mathrm{SH}-1$ (agulhas térmicas duplas), que é inserido na amostra por 2 minutos. Neste trabalho foram realizadas 18 medições de condutividade térmica em cada corpo-de-prova (seis faces) de PUR, conforme Fig. 4, com temperatura média ambiente de $24,37 \pm 0,54{ }^{\circ} \mathrm{C}$.
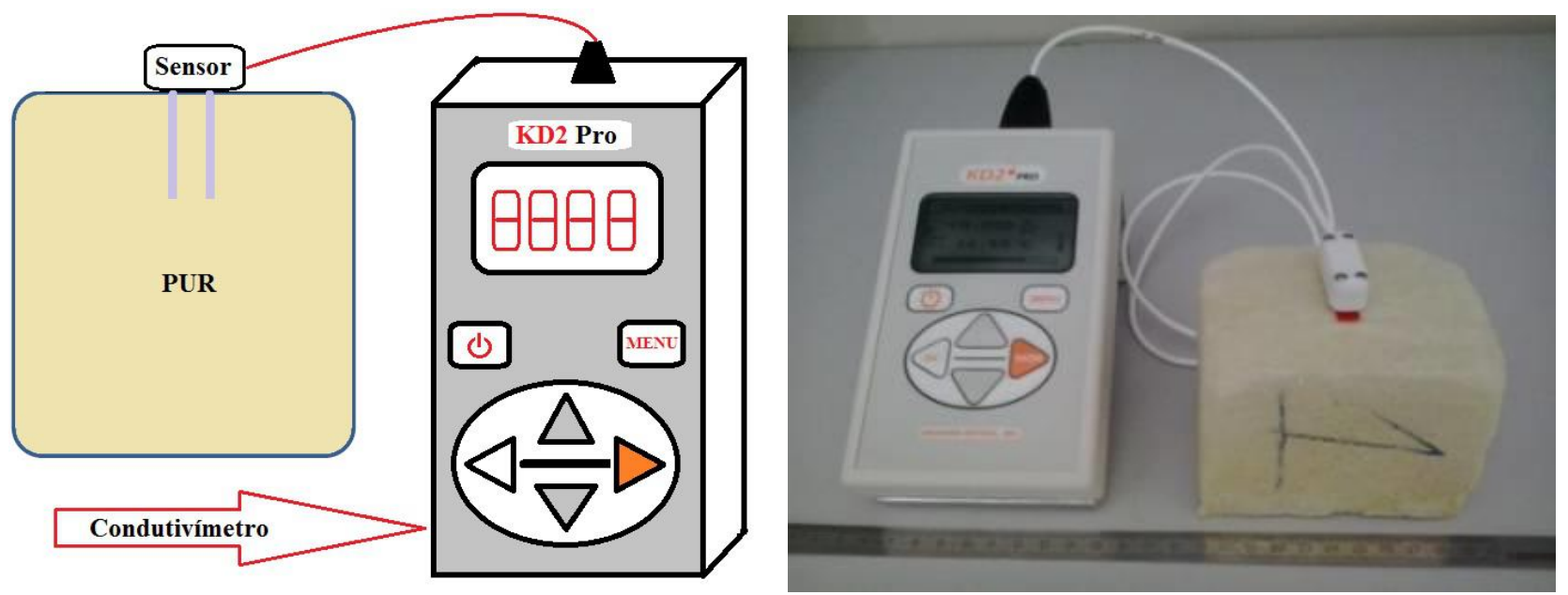

Figura 4 - Análise da condutividade térmica dos corpos de prova usando o condutivímetro KD2 Pro.

\section{RESULTADOS E DISCUSSÃO}

\subsection{Caracterização do PV}

A avaliação da resistência à compressão das PUR com adição de pó de vidro é discutida considerando-se o percentual em massa (5, 10 e $20 \%$ ) de PV e o tamanho de suas partículas $(100 \mu \mathrm{m}$ e $300 \mu \mathrm{m})$. A Figura 5 apresenta dois histogramas gerados, por granulômetro a laser, para avaliação do PV de (a) menor e (b) maior granulometrias. As partículas foram classificadas em diâmetros médios $D_{10}, D_{50}$ e $D_{90}$, cujas medidas são cumulativas a $10 \%, 50 \%$ e $90 \%$ do total de amostras analisadas, respectivamente. 


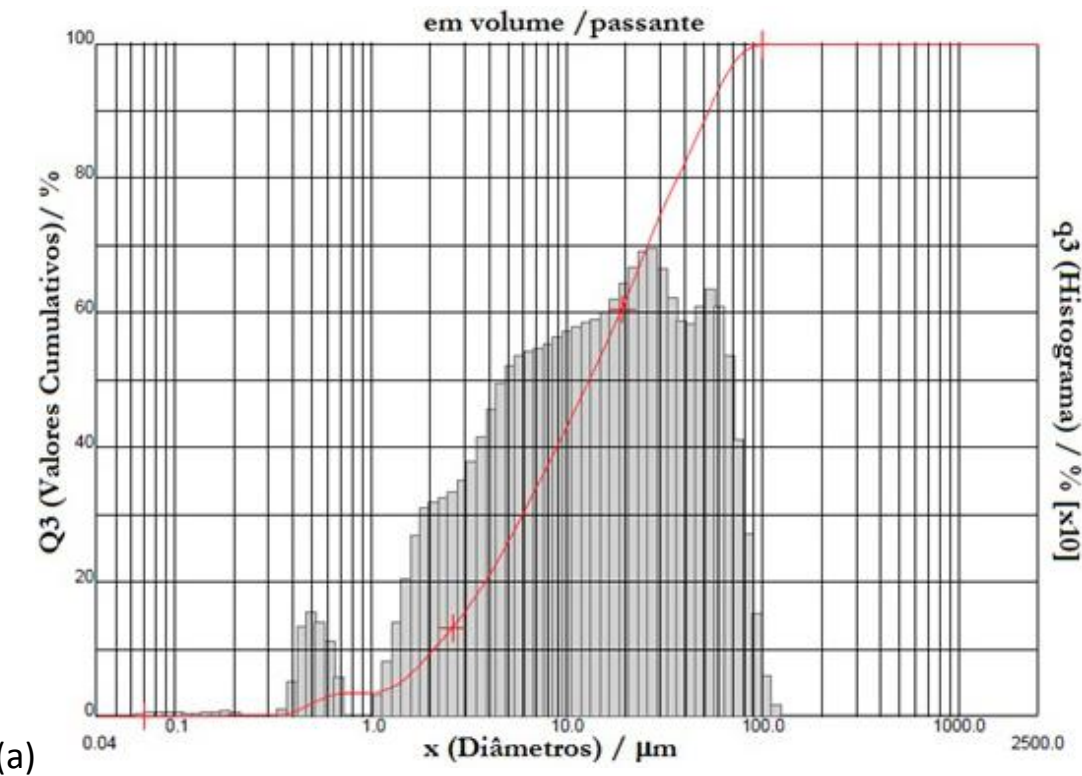

(a)

em volume /passante

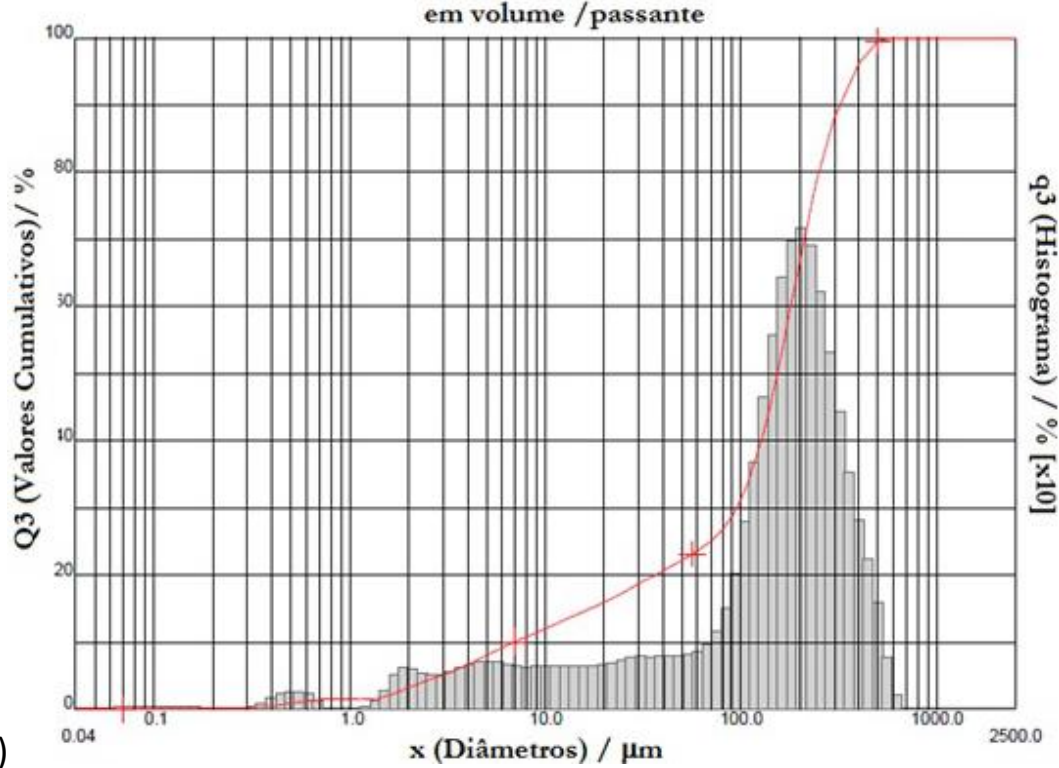

Figura 5 - Granulometria das partículas de PV: partículas até (a) $100 \mu \mathrm{m}$ e (b) $300 \mu \mathrm{m}$.

$\mathrm{Na}$ Figura 5 foi identificada uma maior dispersão entre os valores obtidos para as partículas menores. Isto porque durante o peneiramento foram selecionadas partículas menores que $100 \mu \mathrm{m}$; enquanto que, no caso das partículas maiores, elas ficaram retidas numa faixa entre $100 \mu \mathrm{m}$ e $300 \mu \mathrm{m}$. Pode-se verificar que, com a passagem do pó de vidro em peneiras granulométricas com malha $140(106 \mu \mathrm{m})$ e malha $50(300 \mu \mathrm{m})$, ocorreu a aglomeração destas partículas entre si. Também, durante esta análise, essas partículas dispersaram-se quando em contato com a água; consequentemente foram obtidas partículas menores em maior proporção na amostra. A Figura 5(a) apresenta diâmetro médio de $21 \mu \mathrm{m}$ e tamanhos médios para 10, 50 e $90 \%$ (valores cumulativos) foram $2 \mu \mathrm{m}, 13 \mu \mathrm{m}$ e $53 \mu \mathrm{m}$, respectivamente. Já a Figura $5(\mathrm{~b})$ apresenta um diâmetro médio de $161 \mu \mathrm{m}$, cujos tamanhos médios para $10 \%$, $50 \%$ e $90 \%$ (valores cumulativos) foram $7 \mu \mathrm{m}, 153 \mu \mathrm{m}$ e $320 \mu \mathrm{m}$, respectivamente.

A Figura 6 apresenta o difratograma do PV, na qual se observa a ausência de fases cristalinas, isto é, este espectro representa uma banda típica amorfa em torno de $27^{\circ}$, possivelmente decorrente da presença de sílica na amostra. 


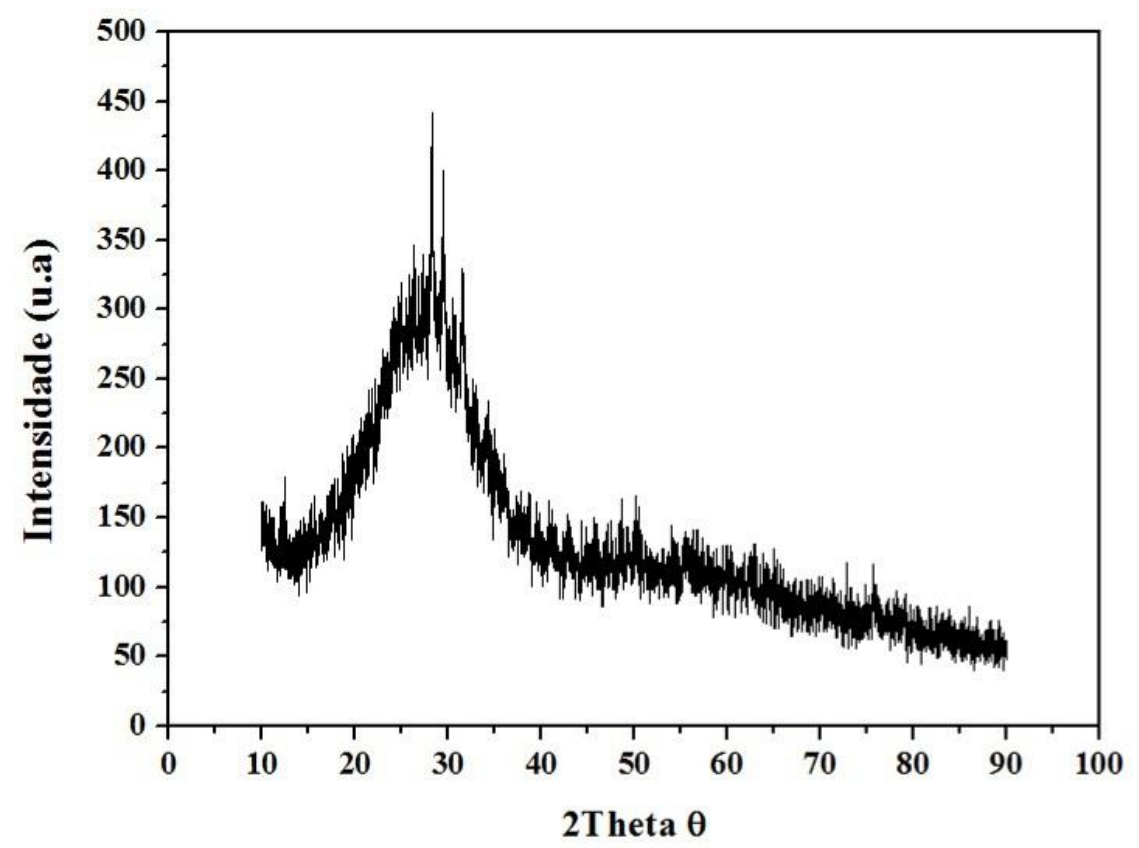

Figura 6 - Difratograma de raios-X do pó de vidro.

Na Tabela 2 são apresentadas as composições químicas em porcentagem (\%) de óxidos presentes no PV proveniente do resíduo da lapidação de vidro, cuja análise foi possível pela técnica de Fluorescência de raios $X$.

Tabela 2: Composição química do pó de vidro (óxidos, \%) obtida por FRX.

\begin{tabular}{c|c}
\hline Óxidos & Percentual (\%) \\
\hline $\mathrm{SiO}_{2}$ & 59,245 \\
\hline $\mathrm{CaO}$ & 20,436 \\
\hline $\mathrm{Na}_{2} \mathrm{O}$ & 10,712 \\
\hline $\mathrm{MgO}$ & 3,969 \\
\hline $\mathrm{ZrO}_{2}$ & 3,411 \\
\hline $\mathrm{Al}_{2} \mathrm{O}_{3}$ & 1,156 \\
\hline $\mathrm{K}_{2} \mathrm{O}$ & 0,551 \\
\hline $\mathrm{Fe}_{2} \mathrm{O}_{3}$ & 0,212 \\
\hline $\mathrm{SO}_{2}$ & 0,203 \\
\hline $\mathrm{IO}_{3}$ & 0,057 \\
\hline $\mathrm{TiO}_{2}$ & 0,024 \\
\hline $\mathrm{SnO}_{2}$ & 0,019
\end{tabular}

Como é observado na Tabela 2, o PV analisado apresenta composição química predominantemente de óxido de silício $\left(\mathrm{SiO}_{2}\right)$, cujo percentual foi de 59,245\%. O óxido de silício é o responsável por formar a rede vítrea. Já o segundo composto mais predominante é o óxido de cálcio ( $\mathrm{CaO})$, principal responsável pela coloração branca e proporciona estabilidade ao vidro contra ataques de agentes atmosféricos. A resistência mecânica do vidro é aumentada em função da presença dos óxidos de alumínio $\left(\mathrm{Al}_{2} \mathrm{O}_{3}\right)$, de zircônio $\left(\mathrm{ZrO}_{2}\right)$ e de magnésio $(\mathrm{MgO})$. Este último garante maior resistência ao vidro para suportar mudanças bruscas de temperatura. Os óxidos dos metais alcalinos e metais alcalinos terrosos (óxidos de sódio, cálcio, e potássio) presentes funcionam como modificadores de rede, sendo responsáveis por romper a estrutura vítrea, diminuindo assim a viscosidade do vidro. 


\subsection{Resistência à Compressão dos Compósitos PUR+PV}

O ensaio de resistência à compressão é útil para estimar a máxima resistência mecânica do corpo-de-prova durante a aplicação de uma força axial compressiva. As Figuras 7 e 8 mostram os gráficos de tensão-deformação para os 35 corpos-de-prova de PUR e compósitos PUR + PV analisados nos seus respectivos percentuais mássicos de PV (5 \%, $10 \%$ e $20 \%$ ) com partículas de $100 \mu \mathrm{m}$ e $300 \mu \mathrm{m}$.
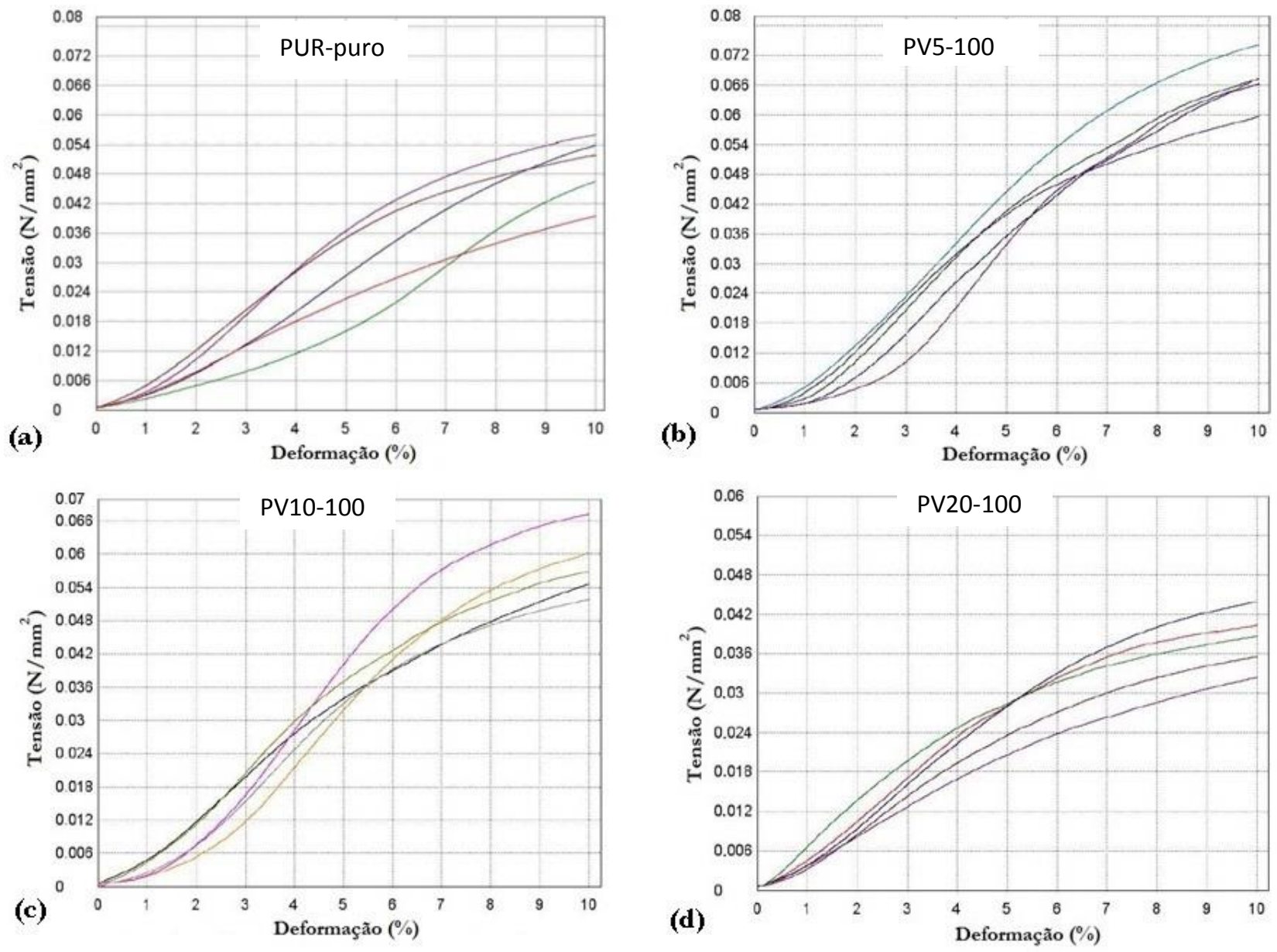

Figura 7 - Gráficos de tensão-deformação de (a) PUR puro e compósitos de PUR com PV (21 $\mu$ m) de percentuais mássicos de (b) $5 \%$, (c) $10 \%$ e (d) $20 \%$.

A Figura 7 apresenta os resultados de resistência à compressão para os corpos-de-prova de PUR + PV de diâmetro médio de $21 \mu \mathrm{m}$ (partículas menores de até $100 \mu \mathrm{m}$ ), enquanto que a Fig. 8 mostra os resultados para os corpos-de-prova de PUR + PV com diâmetro médio de $161 \mu \mathrm{m}$ (partículas maiores de até $300 \mu \mathrm{m}$ ). Os gráficos Fig. 7(a) e Fig. 8(a) referem-se às curvas de tensão-deformação obtidas do ensaio com os corpos-de-prova de PUR-puro (padrão de referência). A Tabela 3 resume os valores médios de resistência à compressão para os corpos-deprova ensaiados. 

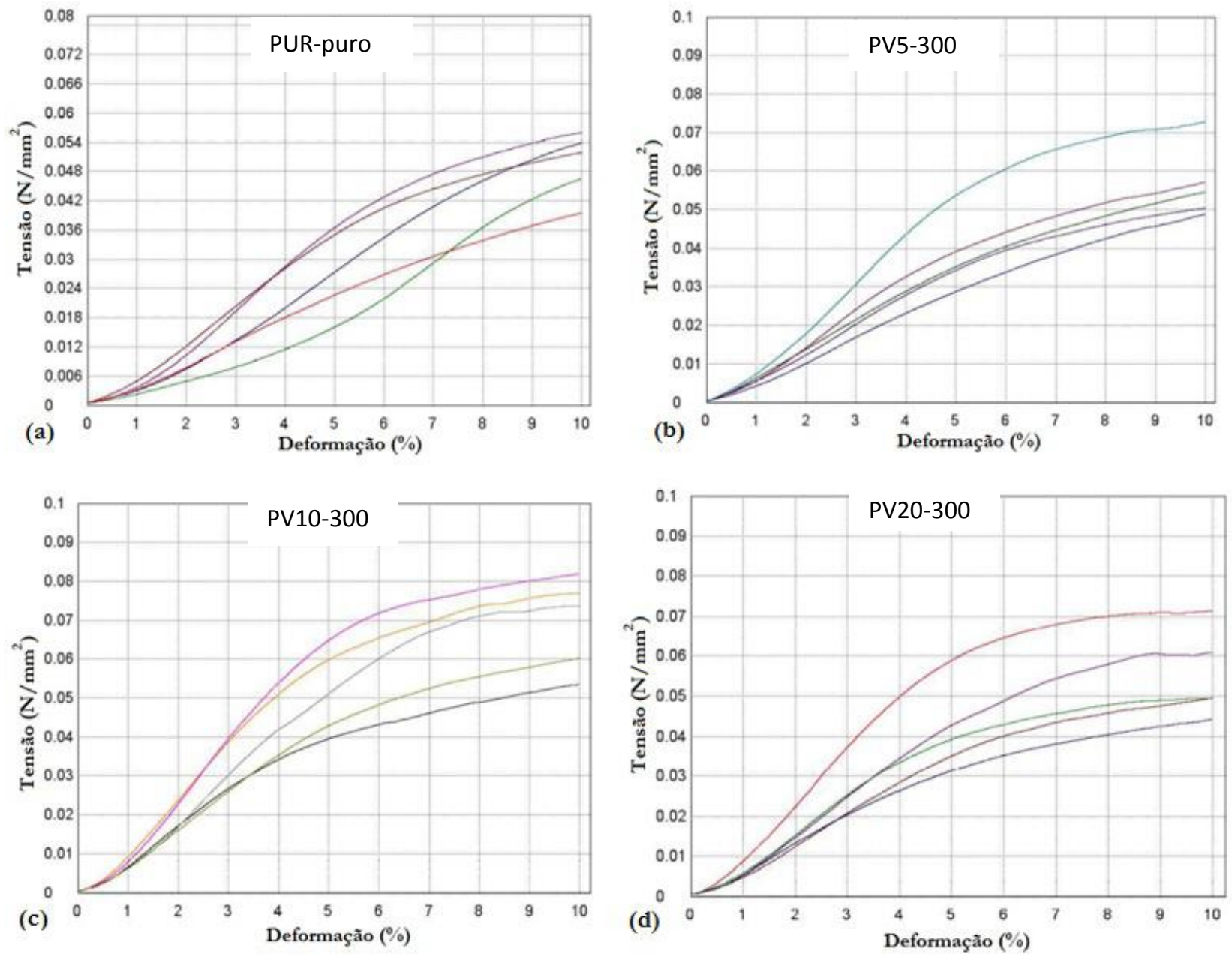

Figura 8 - Gráficos de tensão-deformação do (a) PUR puro e compósitos de PUR com PV (161 $\mu \mathrm{m})$ com percentuais mássicos de (b) $5 \%$, (c) $10 \%$ e (d) $20 \%$.

Como é verificado na Tabela 3, o PV20-100 foi o que apresentou o pior desempenho de resistência mecânica. Os demais corpos-de-prova apresentaram maior resistência à compressão que os de PUR-puro, tanto com a adição de PV $D_{90} 52.80 \mu \mathrm{m}$, quanto de $320 \mu \mathrm{m}$. No entanto os valores de resistência obtidos estão praticamente numa mesma faixa (50 kPa - $69 \mathrm{kPa}$ ).

Tabela 3: Valores médios de resistência à compressão dos corpos-de-prova de PUR + PV.

\begin{tabular}{l|c}
\hline Corpos-de-prova & Valores (kPa) \\
\hline PUR-puro & $50 \pm 07$ \\
\hline PV5-100 & $67 \pm 05$ \\
\hline PV5-300 & $57 \pm 10$ \\
\hline PV10-100 & $58 \pm 06$ \\
\hline PV10-300 & $69 \pm 12$ \\
\hline PV20-100 & $38 \pm 04$ \\
\hline PV20-300 & $55 \pm 11$ \\
\hline
\end{tabular}

Thirumal et al. (2010) adicionaram ao PUR cargas de alumina trihidratada (ATH) e trifenilfosfato (TPP). Eles verificaram que a resistência à compressão aumentou com o uso de $50 \%$ de ATH, entretanto quando era usado PUR preenchida com $50 \%$ de ATH e $10 \%$ de TPP, esta propriedade diminuía de valor devido ao efeito plastificante do TPP com o ATH. Essas literaturas mostram que as iterações entre matriz e cargas nem sempre são positivas. 
Assim, baseado nas Figuras 7 e 8 e na Tabela 3, o PV20-100 apresentou uma diminuição na resistência à compressão em pelo menor $24 \%$ com relação ao PUR-puro. Nota-se que, quando foram usados corpos-de-prova de PUR carregadas com PV de diâmetro médio de $21 \mu \mathrm{m}$, o aumento do conteúdo de PV favoreceu um decréscimo na resistência à compressão. Assim, o PV5-100 exibiu o maior valor de resistência mecânica dentre todos os corpos-de-prova utilizados. No grupo de corpos-de-prova de PUR carregados com PV de diâmetro médio igual a $161 \mu \mathrm{m}$, a adição de 10 \% de PV contribuiu para o aumento na resistência à compressão, ao passo que os demais compósitos forneceram menores elevações nesta propriedade. De uma forma geral, esses resultados inferem que as cargas de PV de $5 \%, 10 \%$ e $20 \%$ agiram como reforço na matriz de PUR, aumentando sua resistência à compressão, e não apenas como carga de enchimento. Isto justiça o uso de PV com o poliuretano, reduzindo o custo final da PUR aplicada para fins de isolação térmica.

\subsection{Massa Específica e Condutividade Térmica dos Compósitos PUR+PV}

As Figuras 9 e 10 mostram os respectivos resultados de massa específica e de condutividade térmica medidos nos corpos-de-prova de PUR carregadas com PV nos percentuais mássicos de $5 \%$, $10 \%$ e $20 \%$. Como já era previsto, a massa específica apresentou aumento proporcional nos compósitos de PUR conforme o teor de PV e do tamanho das partículas presentes na matriz de PUR. Não foi possível notar uma correlação forte entre a densidade e a isolação térmica. Vilar (2007) relatou que a baixa condutividade térmica das espumas PUR resulta da sua baixa densidade e da sua estrutura de células pequenas e fechadas, cheias como agentes de expansão auxiliares (FEAs) como os CFC's, $\mathrm{CO}_{2}, \mathrm{HCFC}$ 's, pentanos, etc. Mas este estudo ficou restrito ao uso de PUR sem adição de carga, assim não foi possível afirmar a influência desta carga sobre a densidade e a isolação térmica. Também se deve considerar que a condutividade térmica final de uma espuma, fator k, é determinada em função das contribuições devidas à: convecção, radiação, condutividade térmica do gás e do polímero e densidade da espuma.

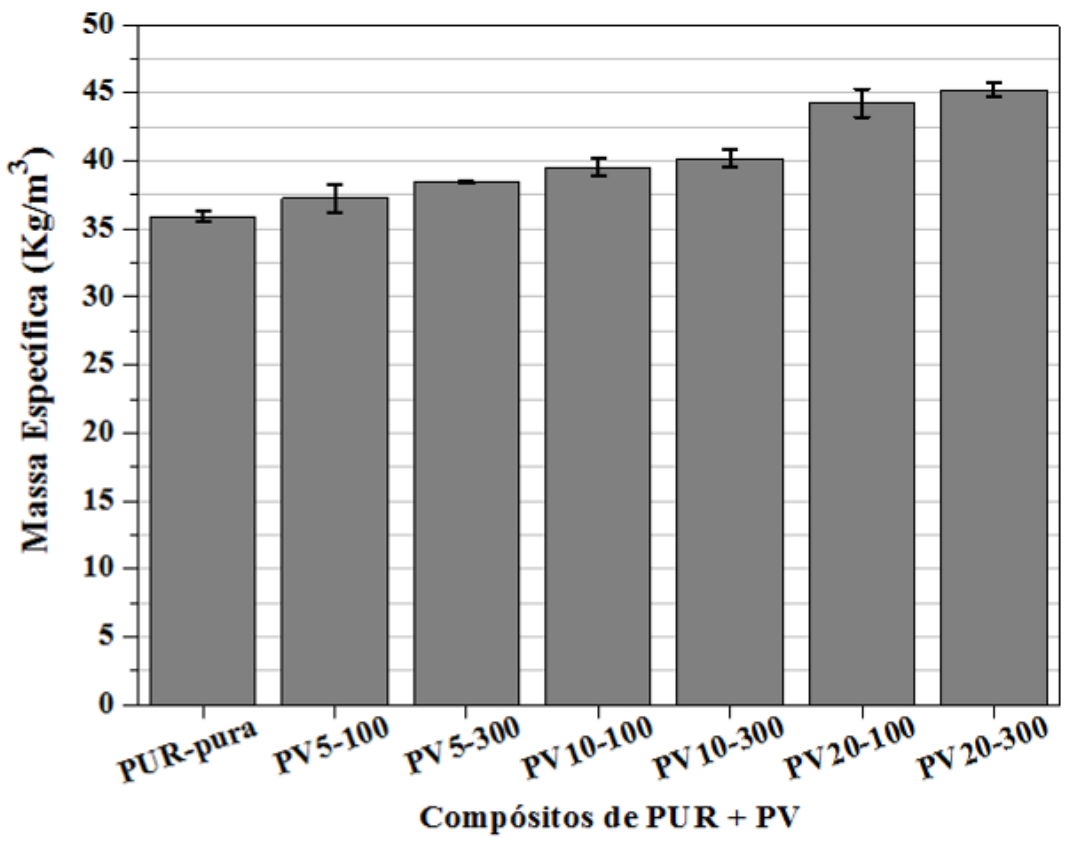

Figura 9 - Massa específica dos corpos-de-prova de PUR + PV. 


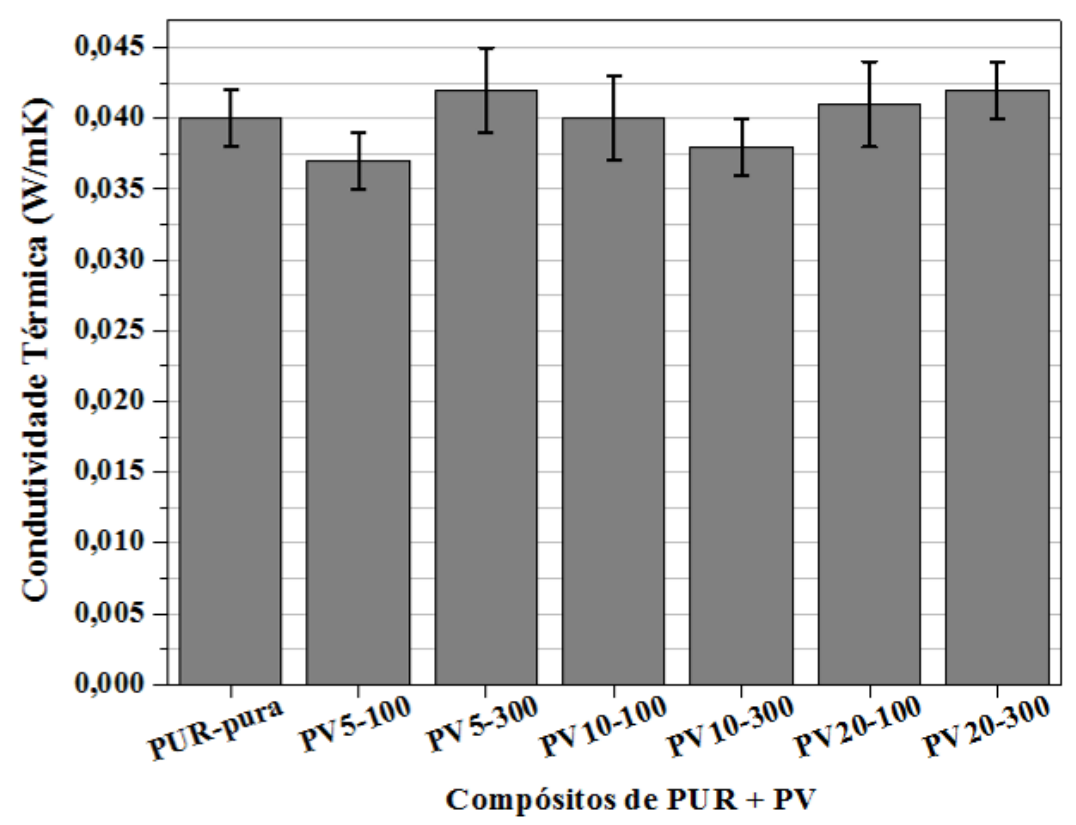

Figura 10 - Condutividade térmica dos corpos-de-prova de PUR + PV.

Nota-se, no gráfico da Figura 10, que o PUR-puro obteve um valor de condutividade térmica de 0,040 W/mK. No entanto os compósitos PV5-100, PV10-300 e PV10-100 apresentaram maiores valores de isolação térmica respectivamente, sendo, portanto, considerados materiais isolantes. Os demais corpos-de-prova destes compósitos apresentaram maiores valores de condutividade térmica que o PUR-puro, isto é, eles obtiveram o pior desempenho de isolação térmica; principalmente os corpos-de-prova com maiores percentuais de PV (PV20-100 e PV20-300). Thirumal et al. (2007) observaram que houve uma redução no isolamento térmico com o aumento dos teores de $\mathrm{SiO}_{2}$ e $\mathrm{CaCO}_{3}$. Isto foi explicado pela forma da estrutura celular gerada aberta e danificada. Eles também notaram que, quando o teor de PV foi usado, a condutividade térmica diminuiu, mas depois elevou-se com o aumento do seu teor.

Esses autores ainda relataram que a estrutura polimérica reticulada com células fechadas apresentou densidades variando de $10 \mathrm{~kg} / \mathrm{m}^{3}$ até $1000 \mathrm{~kg} / \mathrm{m}^{3}$. Segundo ele, os melhores isolantes térmicos tinham densidades entre $28 \mathrm{~kg} / \mathrm{m}^{3}$ até $50 \mathrm{~kg} / \mathrm{m}^{3}$. A característica de isolamento, baseada nesta faixa de densidade, é devido às propriedades de baixa condutividade térmica (fator k) do gás que estão contidos na estrutura celular da espuma. No caso do uso do PV, a sua composição química foi o fator determinante para o aumento da densidade dos compósitos.

Apesar de todos os compósitos de PUR carregados com PV não terem promovido um desempenho superior de isolação térmica em relação ao PUR-puro, o uso de PV pode promover a redução do consumo de energia na obtenção de matérias-primas do PUR-puro, e também pode ser uma alternativa ecológica que garante a reciclagem de materiais destinados a aterros sanitários, como é o caso do vidro.

\section{CONCLUSÃO}

1. Ao se comparar os resultados obtidos com o ensaio de compressão, os corpos-de-prova apresentaram uma resistência à compressão superior em relação aos corpos-de-prova de PUR-puro. Sendo assim, a adição de pós de vidro PV de 5\%, $10 \%$ e $20 \%$ promoveu 
aumento da resistência à compressão na PUR; sendo assim, o PV agiu tanto como carga de reforço, como de enchimento.

2. A influência do tamanho da partícula de pó de vidro contribui na resistência mecânica, sendo que as partículas maiores foram as que mais proporcionaram aumento nos valores de resistência obtidos, com exceção do compósito PV5-300.

3. Dentre os percentuais estudados, a quantidade de $10 \%$ de PV, foi a que mais contribui para o aumento da resistência do composto PUR com valores médios de $58 \mathrm{kPa}$ (PV10-100) e $69 \mathrm{kPa}$ (PV10-300). Enquanto que quando foi usado o percentual de $20 \%$ de PV adicionado ao PUR, sua resistência teve uma ligeira redução, principalmente para o PV20-100, cujo valor foi menor que o do PUR-puro.

4. O aumento do diâmetro das partículas e do teor de PV na matriz da PUR resultou num baixo acréscimo da condutividade térmica, sendo que os corpos-de-prova de PV5-100 com $5 \%$ ofereceram a menor condutividade térmica.

5. De acordo com os resultados da resistência à compressão, massa específica e da condutividade térmica, o uso do PV na matriz do PUR representou uma alternativa econômica e ambientalmente viável.

\section{AGRADECIMENTOS}

Os autores agradecem à CAPES (Coordenação de Aperfeiçoamento de Pessoal em Educação Superior), DVN Vidros LTDA e a todos os técnicos e responsáveis dos laboratórios de Mecânica dos Fluidos, de Máquinas Hidráulicas e Energias Renováveis e de Ensaios Mecânicos do Núcleo Tecnológico Industrial da Universidade Federal do Rio Grande do Norte.

\section{REFERÊNCIAS BIBLIOGRÁFICAS}

1. ABIQUIM, Prêmio de $A B I Q U I M$ de tecnologia, incentivo à pesquisa e inovação. Associação Brasileira da Indústria Química, 2010. Disponível em: <http://www.abiquim.org.br/home/ associacao-brasileira-da-industria-quimica>. Acesso em: 10 fev. 2014.

2. BLEDZKI, A.K., ZHANG, W.Y., CHATE, A. Natural-fiber-reinforced polyurethane microfoams. Composites Science and Technology, v.61, n.16, p.2405-2411, 2001.

3. CANGEMI, J.M. Biodegradação de poliuretano derivado de óleo de mamona. São Carlos, 2006. Tese de doutorado em ciências - química analítica da Universidade de São Paulo, 2006.

4. DEMHARTER, A. Polyurethane rigid foam, a proven thermal insulating material for applications between $+130^{\circ} \mathrm{C}$ and $-196^{\circ} \mathrm{C}$. Cryogenics, v.38, n.1, p.113-117, 1998 .

5. FISCHER, G.R. Gestão de resíduos Industriais. Apostila - GRF Treinamento e Gestão Ambiental. 248p, 2002.

6. KIPPER, L.M., CROSSETTI, G.L, BECKER, R.C. Estudo do aproveitamento do resíduo de espuma rígida de poliuretano no isolamento térmico de produtos de refrigeração - $\mathrm{Na}$ busca da manufatura sustentável. In: 28th Encontro Nacional de Engenharia da Produção. Rio de Janeiro. Trabalhos técnicos. Rio de Janeiro: ABEPRO, 2008. 
7. MELLO, D., SANCHEZ, F.A.L., SANTANA, R.M.C, AMICO, S.C. Estudo da inclusão de PET reciclável como carga de reforço em espumas flexíveis de poliuretana. In: 17ㅇ CBECIMat Congresso Brasileiro de Engenharia e Ciência dos Materiais. Foz do Iguaçu, PR, Brasil, 2006.

8. MENDES, J.U.L. Desenvolvimento de um compósito biodegradável para isolamento térmico. Tese de doutorado apresentada ao Programa de Pós-Graduação em Ciências e Engenharia de Materiais, Universidade Federal do Rio Grande do Norte, 141p, Natal, 2002.

9. OLIVEIRA, M.C.R. Compósito de mamona e resíduo industrial para isolação térmica e absorção sonora. Natal, 2010. Dissertação apresentada ao programa de Pós-Graduação em Engenharia Mecânica da Universidade Federal do Rio Grande do Norte. 77p, 2010.

10. RAPIN, P. Manual do Frio. Editora Hemus, 8o edição, 462p, 2001.

11. SAHA, M.C., KABIR, M.E.; JEELANI, S. Enhancement in thermal and mechanical properties of polyurethane foam infused with nanoparticles. Materials Science and Engineering: A, v.479, n.1-2, p.213-222, 2008.

12. SANTOS, W.J. Caracterização de vidros planos transparentes comerciais. Scientia Plena, v.5, n.2, p.1-5, 2009.

13. SIQUEIRA, L.V.M., STRAMARI, M.R. Adição de poliuretano expandido para a confecção de blocos de concreto leve. Revista Matéria, v.9, n.4, p.399-410, 2004.

14. THIRUMAL, M., KHASTGIR, D., SINGHA, N.K., MANJUNATH, B.S., NAIK, Y.P. Halogen-free flame-retardant rigid polyurethane foams: Effect of alumina trihydrate and triphenylphosphate on the properties of polyurethane foams. Journal of Applied Polymer Science, v.116, n.4, p.2260-2268, 2010.

15. THIRUMAL, M., KHASTGIR, D., SINGHA, N.K., MANJUNATH, B.S., NAIK, Y.P. Effect of expandable graphite on the properties of intumescent flame-retardant polyurethane foam. Journal of Applied Polymer Science, v.110, n.5. p.2586-2594, 2008.

16. THIRUMAL, M., KHASTGIR, D., SINGHA, N.K., MANJUNATH, B.S., NAIK, Y.P. Mechanical, morphological and thermal properties of rigid polyurethane foam: effect of the fillers. Cellular Polymers, v.26, n.4, p.245-259, 2007.

17. TSAI, I.-J., LEI, C.-H., LEE, C.-H., LEE, Y.-C., LOU, C-W., LIN, J.-H. Manufacturing process and property analysis of industrial flame retarded PET fiber and polyurethane composite. Journal of Materials Processing Technology, v.192-193, p.415-421, 2007.

18. TORREIRA, R.P. Isolantes térmicos: frio \& calor. São Paulo: Fulton Editora Técnica LTDA. $1024 p, 1980$.

19. VILAR, W.D. Químicas e tecnologia dos poliuretanos. 3ำed. São Paulo: Editora Pronor, 400p, 2007.

20. VLADIMIROV, V.S., LUKIN, E.S., POPOYA, N.A., ILYUKHIN, MOIZIS, S.E., ARTAMONOV, M.A. New types of light-weight refractory and heat-insulation materials for long-term use at extremely high temperatures. Glass and Ceramics, v.68, n.3-4, p.116-122, 2011.

21. YANG, Z.G., ZHAO, B., QIN, S.L., HU, Z.F., JIN, Z.K., WANG, J.H. Study on the mechanical properties of hybrid reinforced rigid polyurethane composite foam. Journal of Applied Polymer Science, v.92, n.3, p.1493-1500, 2004. 